\title{
Comment on "Time evolution of the fluid flow at the top of the core. Geomagnetic jerks" by M. Le Huy, M. Mandea, J.-L. Le Mouël, and A. Pais
}

\author{
Andrew Jackson \\ Department of Earth Sciences, Leeds University, Leeds LS2 9JT, England
}

(Received July 18, 2000; Accepted August 15, 2000)

In their paper on fluid flow at the core-mantle interface, Le Huy et al. (2000) use the model of Bloxham and Jackson (1992) to derive fluid flows back to 1690. The well-known lack of absolute magnetic intensities prior to 1832 means that it is not possible to uniquely derive the fluid flow prior to this time, as I show below. This means that the results of Le Huy et al.'s study prior to 1832 are nonunique and as such should be viewed with suspicion.

In deriving model ufm2 for the period 1690-1840, Bloxham and Jackson (1992) dealt with the lack of intensities by fixing the dipole to decay from its 1840 value at the rate $15 \mathrm{nT} / \mathrm{yr}$, a technique similar to the one employed previously by Barraclough (1974). The directional data used in the model can determine the morphology of the field, but not its amplitude at any one time.

Let the estimated radial magnetic field model (ufm2) be $B_{r}^{\mathrm{ufm}}$ and the true model be $B_{r}^{\text {true }}$. The two are linked at any time by

$$
B_{r}^{\mathrm{ufm}}(t)=B_{r}^{\text {true }}(t) / \alpha(t)
$$

for any time varying function $\alpha$, since the magnitude of the field cannot be determined.

Under the frozen-flux approximation the induction equation for the true field is

$$
\frac{\partial B_{r}^{\text {true }}}{\partial t}=-\nabla_{h} \cdot\left(\mathbf{u} B_{r}^{\text {true }}\right)
$$

or

$$
\frac{\partial \alpha B_{r}^{\mathrm{ufm}}}{\partial t}=\alpha \frac{\partial B_{r}^{\mathrm{ufm}}}{\partial t}+B_{r}^{\mathrm{ufm}} \frac{\partial \alpha}{\partial t}=-\nabla_{h} \cdot\left(\mathbf{u} \alpha B_{r}^{\mathrm{ufm}}\right)
$$

giving

$$
\frac{\partial B_{r}^{\mathrm{ufm}}}{\partial t}+B_{r}^{\mathrm{ufm}} \frac{\partial \log \alpha}{\partial t}=-\nabla_{h} \cdot\left(\mathbf{u} B_{r}^{\mathrm{ufm}}\right) .
$$

The results depend crucially on the value chosen for $\partial \log$ $\alpha / \partial t$, and there is obviously an infinity of choices of $\alpha(t)$. The model ufm 2 of Bloxham and Jackson (1992) chose $\alpha(t)$ so that $\dot{g}_{1}^{0}$ is constant. Whilst this is a convenient device, this is, as stated above, one arbitrary choice. Moreover, it is very

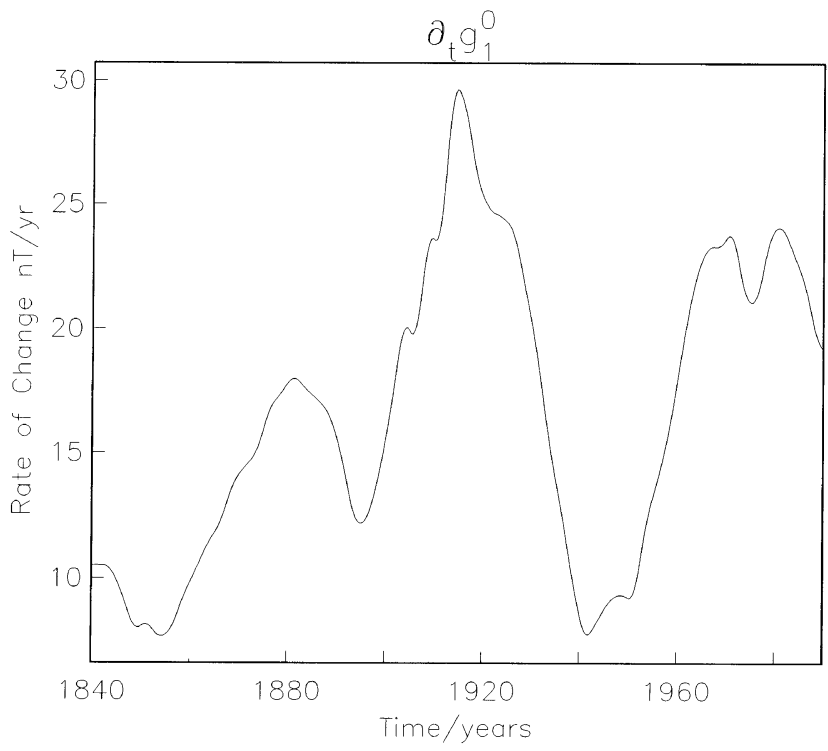

Fig. 1. Rate of change of $g_{1}^{0}$ for the period 1840-1990 from ufm1.

unlikely to give the true underlying secular variation since the rate of change of $g_{1}^{0}$ varied widely during the post-1840 period (see Fig. 1). The use of the frozen flux approximation in generating a field model similar to $u f m 2$ would be a more appropriate and consistent mechanism with which to fix the absolute value of the field $B_{r}$ back in time. Such models are currently under construction.

\section{References}

Barraclough, D. R., Spherical harmonic analyses of the geomagnetic field for eight epochs between 1600 and 1910, Geophys. J. R. Astr. Soc., 36 , 497-513, 1974.

Bloxham, J. and A. Jackson, Time-dependent mapping of the magnetic field at the core-mantle boundary, J. Geophys. Res., 97, 19537-19563, 1992.

Le Huy, M., M. Mandea, J.-L. Le Mouël, and A. Pais, Time evolution of the fluid flow at the top of the core. Geomagnetic jerks, Earth Planets Space, 52, 163-173, 2000.

A. Jackson (e-mail: jackson@earth.leeds.ac.uk) 